\title{
Editorial
}

Nephrology

\section{Polycystic Kidney Disease Progression: Learning from Europe}

\author{
Benjamin D. Cowley Jr \\ Department of Medicine, Nephrology and Hypertension Section, University of Oklahoma Health Sciences Center, \\ Oklahoma City, OK, USA
}

Autosomal dominant polycystic kidney disease (AD$\mathrm{PKD}$ ) is the most common genetic renal disease in humans and arguably the most common potential fatal genetic disease in humans. ADPKD causes the development of cysts derived from renal tubules, progressive enlargement of the kidneys, and end-stage renal disease in approximately $50 \%$ of affected individuals [1]. It can be cause by mutation in the PKD1, PKD2, or GANAB genes [2]. There is great variability in the progression of renal cystic disease and deterioration of renal function in patients with PKD. Some of this is due to mutation in different genes or due to different mutations in the individual genes. However, there is variability in progression even within the same family members who presumably have the same genetic mutation. Thus, other factors must affect disease progression. These factors are thought to be environmental or other genes, so-called modifier genes, in individual patients. This variability takes on new significance when contemplating therapy, which in theory could be lifelong. Individuals with a slowly progressive disease may have limited enthusiasm for therapy with known or unknown side effects.

Much has been learned over the last 35 years regarding the mechanisms of PKD development and progression.
Of note, cyclic $3^{\prime}, 5^{\prime}$ adenosine monophosphate (cAMP) has been shown to promote the proliferation of cells lining cysts and fluid secretion into cysts [1]. In the kidney, cAMP is produced in large part in response to vasopression. A milestone in the approach to ADPKD has been the development of a specific therapy to slow the disease. In the TEMPO 3:4 trial, the vasopressin receptor antagonist, tolvaptan, was shown to slow the growth of cystic kidneys and the deterioration of renal function [3]. On the basis of this trial, tolvaptan was approved as treatment for ADPKD in Japan, Europe, and Canada. Subsequently, tolvaptan again was shown to slow the deterioration of renal function in the REPRISE trial [4]. On the basis of this additional information, tolvaptan was approved as treatment for ADPKD in the United States.

Most drugs have side effects and tolvaptan is no exception. As a result of its mechanism of action, tolvaptan markedly impairs urinary concentrating ability. Patients taking tolvaptan have polyuria, urinary frequency, nocturia, polydipsia, and excessive thirst. In addition, a small percentage of patients develop liver function abnormalities, some of which can be significant, but all of which appear to resolve with drug discontinuation. This rare druginduced liver injury resulted in the institution of a risk

\section{KARGER}

(c) 2018 S. Karger AG, Basel 
evaluation and mitigation strategy. These side effects led to the targeting of ADPKD patients at risk of "rapid progression." Typically, rapid progression of ADPKD is defined as growth of total kidney volume (TKV) $>5 \%$ per year or a fall in estimate glomerular filtration rate of $\geq 5$ $\mathrm{mL} / \mathrm{min} / 1.73 \mathrm{~m}^{2}$ per year.

In anticipation of the use of tolvaptan in ADPKD patients, the European Renal Association-European Dialysis and Transplant Association Working Groups of Inherited Kidney Disorders and European Renal Best Practice (WGIKD/ERBP) developed a consensus algorithm to identify patients at risk for "rapid progression." The WGIKD/ ERBP algorithm involves the sequential assessment of estimate glomerular filtration rate decline, TKV growth, kidney length, age and height-adjusted TKV (htTKV) based on the Mayo ADPKD class [5], PROPKD score [6], and family history of end-stage renal disease at age $\leq 58$.

In Europe, tolvaptan has been used to treat ADPKD for several years, and this experience may provide useful insights. In this issue of AJN, Torra et al. [7] describe their experience in a real-world clinic using the WGIKD/ERBP algorithm to identify ADPKD patients at risk for rapid progression. It should be noted that their clinic is a national referral center for hereditary disease, so their experience may not be typical, but their insights are nevertheless of interest.

The WGIKD/ERBP algorithm appears effective in identifying ADPKD patients at risk of rapid progression; however, it could be argued that it is somewhat cumbersome for use in routine practice. One interesting finding is that the assessment of htTKV and classification according to the methods of Irazabal et al. [5] may be as effective as that of the WGIKD/ERBP algorithm. It should be noted that this method is not designed for use in clinical decision making. However, the U.S. Food and Drug Administration has recognized htTKV as an "enrichment biomarker" designed to identify appropriate ADPKD patients for enrollment in clinical trials of potential therapies.

Tolvaptan is the first therapy specifically designed to slow the progression of any renal disease in recent memory, but it is hoped that it will not be the last. It was developed based on an understanding of disease mechanisms resulting from years of basic and preclinical research. It validates the investments made in renal research over the past several decades. Torra et al. [7] provide valuable insight into how physicians and patients should view the appropriate use of tolvaptan and other ADPKD therapies on the horizon.

\section{Disclosure Statement}

Dr. Benjamin D. Cowley, Jr was on the Independent Data Monitoring Committee for the TEMPO 3:4 and REPRISE trials and in that role was a consultant for Otsuka.

\section{References}

1 Grantham JJ: Clinical practice. Autosomal dominant polycystic kidney disease. N Engl J Med 2008;359:1477-1485.

2 Porath B, Gainullin VG, Cornec-Le Gall E, et al: Mutations in GANAB, encoding the glucosidase II $\alpha$ subunit, cause autosomal-dominant polycystic kidney and liver disease. Am J Hum Genet 2016;98:1193-1207.

3 Torres VE, Chapman AB, Devuyst O, et al: Tolvaptan in patients with autosomal dominant polycystic kidney disease. N Engl J Med 2012;367:2407-2418
4 Torres VE, Chapman AB, Devuyst O, et al: Tolvaptan in later-stage autosomal dominant polycystic kidney disease. N Engl J Med 2017; 377:1930-1942.

5 Irazabal MV, Rangel LJ, Bergstralh EJ, et al: Imaging classification of autosomal dominant polycystic kidney disease: a simple model for selecting patients for clinical trials. J Am Soc Nephrol 2015;26:160-172.
6 Cornec-Le Gall E, Audrezet MP, Rousseau A, et al: The PROPKD score: a new algorithm to predict renal survival in autosomal dominant polycystic kidney disease. J Am Soc Nephrol 2016;27:942-951.

7 Furlano M, Loscos I, Martí T, et al: Autosomal dominant polycystic kidney disease: clinical assessment of rapid progression. Am J Nephrol 2018;48;308-317. 